\section{Gaussian MRF Rotation-Invariant Features for Image Classification}

\author{
Huawu Deng, Member, IEEE, and \\ David A. Clausi, Senior Member, IEEE
}

\begin{abstract}
Features based on Markov random field (MRF) models are sensitive to texture rotation. This paper develops an anisotropic circular Gaussian MRF (ACGMRF) model for retrieving rotation-invariant texture features. To overcome the singularity problem of the least squares estimate method, an approximate least squares estimate method is designed and implemented. Rotation-invariant features are obtained from the ACGMRF model parameters using the discrete Fourier transform. The ACGMRF model is demonstrated to be a statistical improvement over three published methods. The three methods include a Laplacian pyramid, an isotropic circular GMRF (ICGMRF), and gray level cooccurrence probability features.
\end{abstract}

Index Terms-Markov random field (MRF), Gaussian MRF (GMRF) model, isotropic, anisotropic, least squares estimate (LSE), discrete Fourier transform (DFT), rotational invariance, texture analysis, classification.

\section{INTRODUCTION}

A challenging problem in image classification is to extract robust rotation-invariant texture features. A Markov random field (MRF) [1] is a powerful tool to model the probability of spatial interactions in an image and has been extensively applied to extract texture features for image classification. As features based on MRF models are generally rotation-variant [2], the application of MRF models for classifying rotated images is strictly limited.

Some research has alternatively considered the frequency domain to extract rotation-invariant features for image textures. Greenspan et al. [3] applied a set of oriented pyramid filters to an image texture and obtained a set of filtered energies. Porter and Canagarajah [4] removed the $\mathrm{HH}$ wavelet channels and combined the $\mathrm{LH}$ and $\mathrm{HL}$ wavelet channels to obtain rotation-invariant wavelet features. They also used circularly symmetric Gabor filters to extract rotation-invariant features. A further exploration of extracting rotation-invariant features through Gabor filters was done by Haley and Manjunath [5]. Cohen et al. [2] estimated rotation angle and scale parameters based on a GMRF (Gaussian Markov random field) model in the frequency domain. Kashyap and Khotanzad [6] constructed an isotropic circular GMRF (ICGMRF) model to extract rotation-invariant features. The ICGMRF model is defined in a circular neighborhood system. The values of the neighbors which are not located on the image grid are bilinearly interpolated. The values of all equiradius pixels are used to generate only one feature. The ICGMRF model, therefore, discards directional information in the possibly nonisotropic textures [2]. To capture directional information, the ICGMRF model will be extended into a novel anisotropic circular GMRF (ACGMRF) model in this paper. The one-dimensional discrete Fourier transform (1D DFT) will be employed to convert the parameters of the ACGMRF model into a set of rotationinvariant features [3], [7]. Ojala et al. [8] used an occurrence

- The authors are with the Department of Systems Design Engineering, University of Waterloo, 200 University Avenue West, Waterloo, Ontario, Canada N2L 3G1. E-mail: \{h2deng, dclausi\}@engmail.uwaterloo.ca.

Manuscript received 19 Sept. 2002; revised 6 Oct. 2003; accepted 2 Jan. 2004. Recommended for acceptance by $R$. Chellappa.

For information on obtaining reprints of this article, please send e-mail to: tpami@computer.org, and reference IEEECS Log Number 117419. histogram to perform rotation invariant texture classification. Pun and Lee [9] applied a log polar transform to perform texture classification that is both rotation and scale invariant. Some papers that study rotation-invariant texture features perform comparisons with other methods [9], [8], [4]; however, not all papers perform comparisons [2], [3], [5], [6].

As the values of the interpolated neighbors in the ACGMRF model are highly correlated, the parameter estimation is unreliable since a singular or nearly singular matrix may occur when using least squares [10] or maximum likelihood [10]. This paper demonstrates a design of an approximate least squares estimate (ALSE) method to overcome this singularity problem. The rotationinvariant ACGMRF features are shown to be a statistical improvement over rotation-invariant features based on the ICGMRF model, the steerable Laplacian pyramid method [3], and the cooccurrence method [11], when classifying rotated textures.

Here, Section 2 discusses the extension of the ICGMRF model to the ACGMRF model. Section 3 discusses the ALSE method to estimate parameters of the ACGMRF model. Section 4 presents experiments and Section 5 concludes this paper.

\section{Anisotropic Circular Gaussian MRF Model 2.1 Isotropic Circular Gaussian MRF (ICGMRF) Model}

Readers are referred to [1], [12] for details of MRF models, a recognized technique for modelling image textures. In the twodimensional image lattice $\mathrm{S}=\{s=(i, j) \mid 1 \leq i \leq H, 1 \leq j \leq W, i, j$, $H, W \in I\}$ (where $H$ and $W$ are the image height and width in pixels), the pixel values $x=\left\{x_{s} \mid s \in \mathrm{S}\right\}$ are a realization of random variables $\mathrm{X}=\left\{X_{s} \mid s \in \mathrm{S}\right\}$. A typical MRF model is the Gaussian MRF (GMRF) model [1] which is widely used for modeling image textures. The GMRF model is also a stationary noncausal twodimensional autoregressive process which is described by the following difference equation:

$$
x_{s}=\sum_{s+r \in N_{s}} \beta_{r} x_{s+r}+\nu_{s},
$$

where $r$ is the relative position with respect to the central pixel $s$, $N_{s}$ is a neighborhood system centered at $s$, and $\left\{\nu_{s}\right\}$ is a stationary Gaussian noise sequence with a zero mean and a known autocorrelation [1]. $\beta_{r}$ is the parameter describing directional information between pixels $x_{s+r}$ and $x_{s}$. All $\beta_{r}$ in the neighborhood system $N_{s}$ form the vector parameter $\beta=\left\{\beta_{r} \mid s+r \in N_{s}\right\}$.

The property of the neighborhood system $N_{s}$ is determined by its order and structure. The order of $N_{s}$ determines the spatial range of the neighborhood. The structure of the neighborhood system $N_{s}$ determines the spatial distribution of neighbors in the neighborhood. For the parameter vector $\beta$, its dimensionality and inherent directionality are therefore dependent on the order $n$ together with the structure of the neighborhood system $N_{s}$.

A rectangular grid is typically used as the structure of the neighborhood system $N_{s}$ in a GMRF model [1], [10], [12]. Here, such a neighborhood system is referred to as a rectangular neighborhood (RN) system. Fig. 1a shows an RN system in different orders. The shape of the RN system changes when it is rotated by any angle except multiples of $\frac{\pi}{2}$ radians. Each rotation of an RN system will therefore generate a different spatial distribution of neighbors.

Kashyap and Khotanzad [6] proposed for the GMRF model a circular neighborhood $(\mathrm{CN})$ system (shown in Fig. 1b) which can achieve a rotation-invariant spatial distribution of neighbors. All neighbors in a $\mathrm{CN}$ system are located on only a single circle. Values of the neighbors are estimated using the bilinear interpolation 


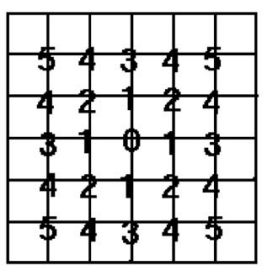

(a)

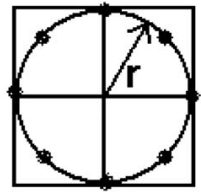

(b)

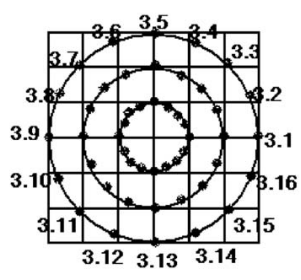

(c)
Fig. 1. (a) The fifth-order rectangular neighborhood system. (b) The first-order circular neighborhood system. $r=1$ is the radius of the circle. (c) The third-order and 16-orientation circular neighborhood system. The number $3 . x$ denotes the $x$ th neighbor on the third concentric circle.

method. Kashyap and Khotanzad further restricted the parameter $\beta_{r}$ to be the same value if $s+r$ is located on the same concentric circle. Equation (1) is changed to:

$$
x_{s}=\sum_{k=1}^{n} \beta_{k}\left[\sum_{|r|=k, s+r \in N_{s}} x_{r}\right]+\nu_{s} .
$$

This GMRF model is referred to as an isotropic circular GMRF (ICGMRF) model in this paper.

Although the features based on the vector parameter $\beta=$ $\left\{\beta_{k}, k=1, \ldots, n\right\}$ are rotation-invariant, their limitation of modelling textures is obvious as textures do not necessarily have isotropic neighbors but anisotropic neighbors that can be reflected as directional information [2].

\subsection{Anisotropic Circular Gaussian MRF (ACGMRF) Model}

Definition 1. An $n$th order circular neighborhood (CN) system is $C N_{s}^{n}=\{s+r|| r \mid=k, 0<k \leq n, k \in I\}$. All neighbors on the same concentric circle are evenly distributed, and the number of neighbors on different circles is the same.

According to this definition, the $\mathrm{CN}$ system used by Kashyap and Khotanzad [6] is the first order $\mathrm{CN}$ system $C N_{s}^{1}$. An angle interval between two nearest neighbors on the same concentric circle is defined as $\theta=\frac{2 \pi}{\tau}$, where $\tau$ is the number of neighbors on one concentric circle in the $\mathrm{CN}$ system. The third-order $\mathrm{CN}$ system with 16 orientations is shown in Fig. 1c. It can be seen that a $C N_{s}^{3}$ has a similar spatial distribution of neighbors as the ninth-order $\mathrm{RN}$ system. The GMRF model defined in the $n$th order $\mathrm{CN}$ system $C N_{s}^{n}$ has the following difference equation:

$$
x_{s}=\sum_{s+r \in C N_{s}^{n}} \beta_{r} x_{s+r}+\nu_{s}
$$

As the parameter $\beta_{r}$ may be different for different circular neighbors, this model is referred to as an anisotropic circular GMRF (ACGMRF) model. Note that this equation is still a function of the order $n$ in the CN system.

\section{Parameter Estimation}

\subsection{Approximate Least Squares Estimate}

Due to its computation efficiency, the least squares estimate (LSE) method has been commonly accepted to estimate the parameters of GMRF models [10]. Define a quadratic difference $Q$ between the central pixel $x_{s}$ and its neighbors in the ACGMRF model:

$$
Q=\sum_{s}\left(x_{s}-\sum_{s+r \in C N_{s}^{n}} \beta_{r} x_{s+r}\right)^{2} .
$$

The least squares estimate of $\beta_{r}$ is [10]:

$$
\beta=\left[\sum_{s} Z_{s} Z_{s}^{T}\right]^{-1}\left[\sum_{s} Z_{s} x_{s}\right],
$$

where $Z_{s}=\operatorname{col}\left\{x_{s+r} \mid s+r \in C N_{s}^{n}\right\}$.

This solution may encounter a singularity problem when $\tau \times n$ is larger than the number of neighbors in the rectangular grid. This is because the interpolated values of the neighbors are highly correlated. The estimate is unreliable when encountering a singular or near singular matrix. Using uncorrelated data can overcome the singularity problem. For this purpose, the parameters of the ACGMRF model can be divided into a number of groups and then estimated separately.

Denote the set of all parameters of the $n$th order ACGMRF model by $\beta=\left\{\beta_{r} \mid s+r \in C N_{s}^{n}, \forall s\right\} . \quad \beta$ can be divided into $m$ groups: $\beta^{1}, \beta^{2}, \cdots, \beta^{m}$. Each group $\beta^{k}$ describes a corresponding group of neighbors in $C N_{s}^{n}$, denoted by $\left(C N_{s}^{n}\right)^{k}$. The parameter groups $\beta^{1}, \beta^{2}, \cdots, \beta^{m}$ should satisfy the following conditions:

1. $\beta^{k}=\left\{\beta_{r}^{k} \mid \beta_{r}^{k}=\beta_{r}, s+r \in\left(C N_{s}^{n}\right)^{k}\right\}$, where $1 \leq k \leq m$ and $k \in I$.

2. $\beta^{k} \neq \emptyset$ and $\beta^{k} \bigcap \beta^{k^{\prime}}=\emptyset$, for $1 \leq k, k^{\prime} \leq m$ and $k \neq k^{\prime}$.

3. $\beta^{1} \cup \beta^{2} \cup \cdots \cup \beta^{m}=\beta$.

The groups of pixel values $x_{C N_{s}^{n}}=\left\{x_{s+r} \mid s+r \in C N_{s}^{n}\right\}$ corresponding to $\beta^{1}, \beta^{2}, \cdots, \beta^{m}$ are denoted by $x_{C N_{s}^{n}}^{1}, x_{C N_{s}^{n}}^{2}, \cdots, x_{C N_{s}^{n}}^{m}$, where $x_{C N^{n}}^{k}=\left\{x_{s+r}^{k} \mid x_{s+r}^{k}=x_{s+r}, s+r \in\left(C N_{s}^{n}\right)^{k}\right\}$. Based on taking partial derivative of the quadratic difference $Q$ (for succinctness, the details are not shown), two approximate least squares estimation method can be obtained. The first approximate least squares estimate (ALSE) method can be designed as:

$$
\beta^{k}=\left[\sum_{s} Z_{s}^{k}\left(Z_{s}^{k}\right)^{T}\right]^{-1}\left[\sum_{s} Z_{s}^{k} x_{s}\right],
$$

where $Z_{s}^{k}=\operatorname{col}\left\{x_{s+r} \mid s+r \in\left(C N_{s}^{n}\right)^{k}\right\}$ and $1 \leq k \leq m$. The second approximate least squares estimate (ALSE) method can be designed as:

1. For estimating the first group of parameters $\beta^{1}$,

$$
\beta^{1}=\left[\sum_{s} Z_{s}^{1}\left(Z_{s}^{1}\right)^{T}\right]^{-1}\left[\sum_{s} Z_{s}^{1} x_{s}\right] .
$$

2. For estimating the $k$ th group of parameters $\beta^{k}$, where $1<k \leq m$,

$$
\beta^{k}=\left[\sum_{s} Z_{s}^{k}\left(Z_{s}^{k}\right)^{T}\right]^{-1}\left[\sum_{s} Z_{s}^{k}\left(x_{s}^{\prime}+x_{s}\right)\right],
$$

where $x_{s}^{\prime}=\sum_{k^{\prime}=1}^{k-1}\left(\frac{1}{m} x_{s}-\sum_{\beta^{k^{\prime}}} \beta_{r}^{k^{\prime}} x_{s+r}^{k^{\prime}}\right)$.

Let $\hat{\beta}_{0}$ denote the set of parameters estimated by the LSE method, $\hat{\beta}_{1}$ the set of parameters estimated by the first ALSE method, and $\hat{\beta}_{2}$ the set of parameters estimated by the second ALSE method. Three quadratic differences can be obtained: $Q_{0}$ by $\hat{\beta}_{0}, Q_{1}$ by $\hat{\beta}_{1}$, and $Q_{2}$ by $\hat{\beta}_{2}$. The smaller the difference $\left|Q_{i}-Q_{0}\right|$ $(i \in\{1,2\})$ the better the ALSE solution approximates the LSE solution.

An example for estimating parameters of the linear system $y=$ $a x_{1}+b x_{2}$ is given here. The parameters $(a, b)$ are to be estimated and a total of $1,024(=32 \times 32)$ sets of $y, x_{1}, x_{2}$ are generated randomly. Then, the LSE method and both ALSE methods are applied. The differences $Q_{0}, Q_{1}$, and $Q_{2}$ are computed with the corresponding estimated parameters. The above experiment is repeated 200 times to obtain 200 sets of $Q_{0}, Q_{1}$, and $Q_{2}$ plotted in Fig. 2. $Q_{2}$ varies closely to $Q_{0}$, while $Q_{1}$ has a larger deviation from $Q_{0}$. This observation is also 


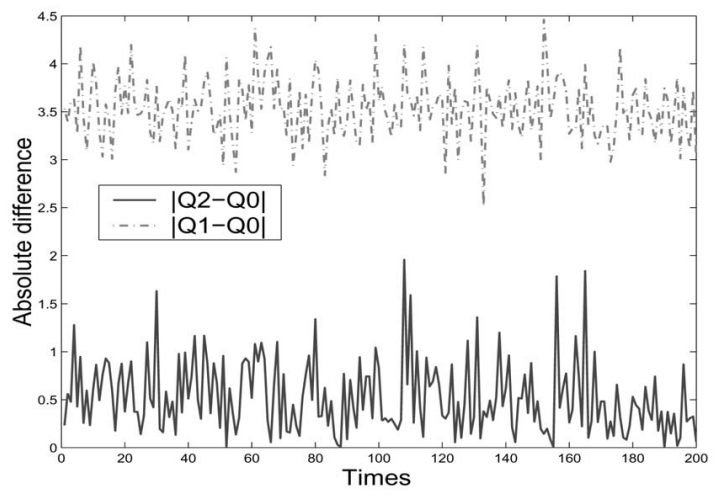

Fig. 2. The comparison between the LSE method and the two ALSE methods. $Q 0$, $Q 1$, and $Q 2$ are the quadratic differences obtained by the LSE, first ALSE, and second ALSE methods, respectively.

supported by two indicators: $p_{1}=\frac{\left|\operatorname{mean}\left(Q_{1}\right)-\operatorname{mean}\left(Q_{0}\right)\right|}{\operatorname{mean}\left(Q_{0}\right)} \times 100$ percent and $p_{2}=\frac{\mid \text { mean }\left(Q_{2}\right)-\text { mean }\left(Q_{0}\right) \mid}{\operatorname{mean}\left(Q_{0}\right)} \times 100$ percent, where mean $\left(Q_{i}\right)$ is the mean of the 200 sets of $Q_{i}$. Here, $p_{1}=2.91$ percent and $p_{2}=0.43$ percent. Therefore, the second ALSE method is used for texture feature extraction in this paper.

The following rules are adopted in this paper to divide parameters of the ACGMRF model into different groups for the ALSE method.

1, The parameters for the neighbors on different concentric circles should be divided into different groups.

2, A basic number of parameters for the $n$th concentric circle is set to the number of pixels on the border of the nearest rectangular grid. When the number of parameters on the $n$th concentric circle exceeds the basic number of the $n$th concentric circle, these parameters should be separated into different groups.

3, The angle intervals $\theta$ between the nearest neighbors in a group should be consistent.

4, The parameters on a lower-order concentric circle should be estimated before those on a higher-order concentric circle.

\subsection{Rotation-Invariant Features}

The parameters of the ACGMRF model form a one-dimensional (1D) vector. A rotation of the circular neighborhood is therefore equivalent to shifting the 1D parameter vector [7]. The 1D discrete Fourier transform (1D DFT) can be applied to the 1D parameter vector and obtain a magnitude vector of the DFT coefficients. The magnitude vector corresponding to a shifted vector remains unchanged with respect to the magnitude vector corresponding to the original vector. This DFT-based magnitude vector is therefore a set of rotation-invariant features. Note that the number of nonzero and nonredundant components in the magnitude vector is much smaller than the total dimension of the magnitude vector. For a symmetric ACGMRF model (which is implemented in experiments of this paper), the number of nonzero and nonredundant components of the magnitude vector is not greater than $\frac{n \times \tau}{4}+1$, as the values of the $1 \mathrm{D}$ parameter vector are real and symmetric.

\section{EXPERIMENTAL RESULTS}

\subsection{Synthesis of Rotated Image Textures}

There are two objectives for this texture synthesis experiment. One is to verify that the ALSE method is able to properly estimate the parameters of the ACGMRF model. Another is to verify that a rotation of the circular neighborhood system, which corresponds to a shift of the parameter vector, will accordingly generate a rotated image texture.

The raffia texture (D084) in the Brodatz database [13] is selected as the original texture. The ALSE method is used to estimate a set of parameters of the third-order and 16-orientation ACGMRF model. Five angles $\left(0, \frac{\pi}{8}, \frac{\pi}{4}, \frac{3 \pi}{8}\right.$, and $\frac{\pi}{2}$ radians) are used to rotate the circular neighborhood system in the counterclockwise direction, and five sets of shifted parameter vectors for each rotated texture are generated, accordingly. The Metropolis sampling method [1] is employed for texture synthesis. The synthesized rotated textures in Figs. 3c, 3d, 3e, and $3 \mathrm{f}$ display the rotated directional information relative to the original texture.

\subsection{Classification Methodology}

The classification experiments are performed in a supervised manner. All image texture samples are placed into separate training and testing sets. A set of rotation-invariant features based on the ACGMRF model is extracted from each image texture sample, denoted by $f_{j}=\left\{f_{j k} \mid 1 \leq k \leq \frac{\tau \times n}{4}+1\right\}$ for the $j$ th sample. The mean of the training class is denoted by $\mu_{i}=\left\{\mu_{i k}=\right.$ $\left.\frac{1}{\operatorname{dim}\left(f^{i}\right)} \sum_{f_{j} \in f^{i}} f_{j k} \mid 1 \leq k \leq \frac{\tau \times n}{4}+1\right\}$ for the $i$ th texture class whose feature set is $f^{i}$. The distance used in [6] is used in this paper as the metric to measure the feature distance:

$$
\hat{i}=\arg _{i} \min \left\{D\left(f_{j}, \mu_{i}\right), i=1,2, \cdots, n\right\},
$$

where $D\left(f_{j}, \mu_{i}\right)=\sum_{k=1}^{\frac{7 \times n}{4}+1}\left|\frac{f_{j k}-\mu_{i k}}{\sigma_{k}}\right|$, and $\sigma_{k}=s t d\left\{f_{j k}\right.$, for all $\left.j\right\}$. An error matrix is produced when classifying the test data and Kappa $(\kappa)$ coefficients and associated confidence intervals $(\sigma)$ are determined [14].

\subsection{Classification of Brodatz Textures}

Brodatz textures [13] are commonly used as test data for generic texture interpretation research. Each Brodatz sample is assumed to contain only one class. Twelve Brodatz textures are selected for classification (Fig. 4). Each Brodatz texture has $256 \times 256$ pixels. These Brodatz textures include regular textures, i.e., D006, D011, D019, D021, D052, D079, D095, and nonregular textures, i.e., D029, D036, D037, D084, D087.

Nonrotated images are used for the training data. Test data is obtained by rotating the images over eleven angles $\left(\frac{\pi}{12}, \frac{\pi}{6}, \frac{\pi}{4}, \frac{\pi}{3}, \frac{5 \pi}{12}, \frac{\pi}{2}\right.$, $\frac{7 \pi}{12}, \frac{2 \pi}{3}, \frac{3 \pi}{4}, \frac{5 \pi}{6}$, and $\frac{11 \pi}{12}$ radians). Only the central $128 \times 128$ regions are used to avoid boundary problems when rotating. Each $128 \times 128$ rotated image is separated into sixteen $32 \times 32$ nonoverlapping subimages to represent the texture samples. Then, a set of rotationinvariant features is extracted from each subimage modeled by the

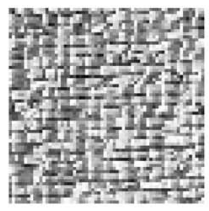

(a)

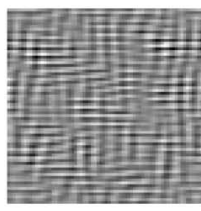

(b)

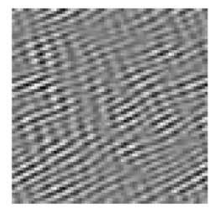

(c)

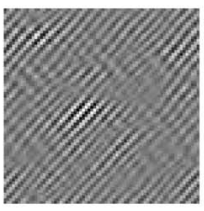

(d)

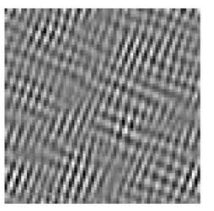

(e)

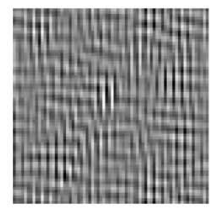

(f)

Fig. 3. Texture synthesis based on the ACGMRF model by rotating its CN system. (a) Original texture (D084); (b), (c), (d), (e), and (f) are synthesized textures with the parameters rotated $0, \frac{\pi}{8}, \frac{\pi}{4}, \frac{3 \pi}{8}$, and $\frac{\pi}{2}$ radians, respectively. 

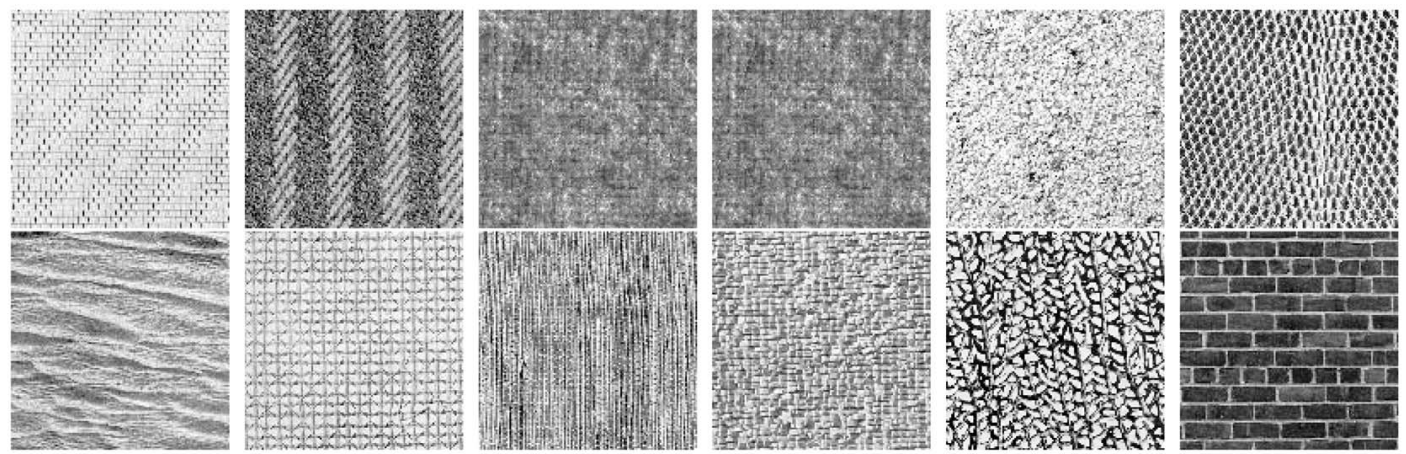

Fig. 4. Twelve Brodatz image textures. First row from left to right: D006 (wire), D011 (woolen cloth), D019 (woven cloth), D021 (French canvas), D029 (sand), D036 (lizard cloth); second row from left to right: D037 (water), D052 (oriental shaw cloth), D079 (oriented grass-fiber cloth), D084 (raffia), D087 (fossilized sea fan), D095 (brick wall).

TABLE 1

Classification Rates (\%) over 12 Brodatz Textures by the Rotation-Invariant Features of the ACGMRF Model, the ICGMRF Model, the Steerable Laplacian Pyramid (SLP) Method, and the GLCP Method, and Corresponding Kappa Coefficients $(\kappa)(\sigma)$

\begin{tabular}{c|c|r|r|r|r}
\hline & Texture Name & ACGMRF & ICGMRF & SLP & GLCP \\
\hline & D006 & 82.3 & 77.1 & 73.4 & 66.7 \\
& D011 & 81.3 & 56.3 & 93.8 & 91.7 \\
& D019 & 87.5 & 54.2 & 97.9 & 50.0 \\
& D021 & 81.3 & 61.5 & 68.2 & 50.0 \\
Classification & D029 & 75.0 & 55.2 & 86.5 & 100.0 \\
Rate (\%) & D036 & 84.3 & 44.8 & 66.7 & 83.3 \\
& D037 & 88.5 & 34.4 & 39.1 & 83.3 \\
& D052 & 78.1 & 37.5 & 43.2 & 58.3 \\
& D079 & 82.3 & 53.2 & 61.5 & 16.7 \\
& D084 & 74.0 & 54.2 & 81.8 & 33.3 \\
& D087 & 82.3 & 44.8 & 73.0 & 100.0 \\
& D095 & 88.5 & 65.6 & 33.3 & 33.3 \\
\cline { 2 - 6 } & Average & 82.1 & 53.2 & 68.2 & 63.9 \\
\hline Evaluation of & $\kappa$ & 0.799 & 0.524 & 0.653 & 0.606 \\
Error Matrix & $\sigma$ & 0.0094 & 0.0121 & 0.01059 & 0.0109 \\
\hline
\end{tabular}

third-order and 24-orientation ACGMRF model. By the classification scheme in (9), the test subimages will be classified into one of the 12 texture classes.

The ACGMRF model is compared to three other published methods that extract rotation-invariant texture features. First, the third-order ICGMRF model features are used as discussed earlier. Second, rotation-invariant features are produced using the method proposed by Greenspan et al. [3]. Three scales are used with four orientations per scale to retrieve steerable Laplacian pyramid (SLP) features [3]. Third, the gray level cooccurrence probability (GLCP) texture features [11] are used. The GLCP features are generated by setting three displacements $(1,2,3)$, four orientations $\left(0, \frac{\pi}{4}, \frac{\pi}{2}, \frac{3 \pi}{4}\right.$ radians), and three statistics (entropy, contrast, and correlation) using 64 quantized gray levels and a $9 \times 9$ window [15]. The 1-DFT method is then applied to convert the GLCP features to be rotationinvariant.

The results listed in Table 1 show the rotation-invariant features of the ACGMRF model achieve the highest overall classification rate among all four methods. Although the SLP method can classify part of the data set at more than 95 pecent, it fails to classify textures that tend to be nonstationary such as those in
D021, D036, D037, D052, D079, and D095. The GLCP method shows strong ability for classifying textures in D011, D029, and D087, but is not successful for classifying those textures in D019, D021, D052, D079, D084, and D095. This is because the GLCP method is relatively weak for capturing information for a regular texture which has a strong and dominant response in certain frequencies. Comparative statistical testing of the error matrices demonstrate that the ACGMRF model is a significant improvement over each of the ICGMRF model, the SLP method, and the GLCP method.

As cited in the bibliography, there exist other research papers that have studied rotation-invariant texture classification. There is no attempt here to directly compare these classification results to the classification results in these studies since this would not be scientifically sound. Each paper uses different data sets, different training/testing samples, a different number of training/testing samples, different classification methods, different sized windows, etc. As such, interpaper comparisons based on percentage classification accuracy are not suitable. It is noted that there is a tendency for other papers to use window sizes larger than $32 \times 32$. A $64 \times 64$ window contains 400 percent of the spatial information 
compared to a $32 \times 32$ window and is thus expected to generate higher classification accuracies. When the same ACGMRF method is applied to $64 \times 64$ samples of the same data set, the classification rate was indeed higher (95.8 percent versus 82.1 percent).

\section{Conclusion}

An anisotropic circular GMRF (ACGMRF) model is developed by extending the isotropic circular GMRF (ICGMRF) model to model textures with directional information. To overcome the singularity problem for the least squares estimate method, an approximate least squares estimate method is developed and successfully implemented in the paper. By using the one-dimensional DFT, the parameters of the ACGMRF model can be converted into a set of rotation-invariant features. Experimental results indicate that a significant improvement of classification accuracy is achieved by the ACGMRF model with respect to the ICGMRF model and two other published methods. It is recommended that the ACGMRF model be used for feature extraction of anisotropic textures.

\section{ACKNOWLEDGMENTS}

The authors thank GEOIDE (http://www.geoide.ulaval.ca/) and CRYSYS (http://www.crysys.ca) for financial support of this project.

\section{REFERENCES}

[1] S.Z. Li, Markov Random Field Modeling in Computer Vision. New York: Springer-Verlag, 2001.

[2] F.S. Cohen, Z.G. Fan, and M.A. Patel, "Classification of Rotated and Scaled Textured Images Using Gaussian Markov Random Field Models," IEEE Trans. Pattern Analysis and Machine Intelligence, vol. 13, no. 2, pp. 192-202, Feb. 1991.

[3] H. Greenspan, S. Belongie, R. Goodman, and P. Perona, "Rotation Invariant Texture Recognition Using a Steerable Pyramid," Proc. 12th IAPR Int'l Conf. Pattern Recognition, vol. 2, pp. 162-167, 1994.

[4] R. Porter and N. Canagarajah, "Robust Rotation-Invariant Texture Classification: Wavelet, Gabor Filter and GMRF Based Schemes," IEE Proc. Conf. Vision, Image, and Signal Processing, vol. 144, no. 3, pp. 180-188, 1997.

[5] G.M. Haley and B.S. Manjunath, "Rotation-Invariant Texture Classification Using a Complete Space-Frequency Model," IEEE Trans. Image Processing, vol. 8, no. 2, pp. 255-269, 1999.

[6] R.L. Kashyap and A. Khotanzad, "A Model-Based Method for Rotation Invariant Texture Classification," IEEE Trans. Pattern Analysis and Machine Intelligence, vol. 8, no. 7, pp. 472-481, July 1986.

[7] H. Arof and F. Deravi, "Circular Neighborhood and 1-D DFT Features for Texture Classification and Segmentation," IEE Proc. Conf. Vision, Image, and Signal Processing, vol. 145, no. 3, pp. 167-172, 1998.

[8] T. Ojala, M. Pietikainen, and T. Maenpaa, "Multiresolution Gray-Scale and Rotation Invariant Texture Classification with Local Binary Patterns," IEEE Trans. Pattern Analysis and Machine Intelligence, vol. 24, no. 7, pp. 971-987, July 2002.

[9] C.-M. Pun and M.-C. Lee, "Log-Polar Wavelet Energy Signatures for Rotation and Scale Invariant Texture Classification," IEEE Trans. Pattern Analysis and Machine Intelligence, vol. 25, no. 5, pp. 590-603, May 2003.

[10] R.L. Kashyap and R. Chellapa, "Estimation and Choice of Neighbors in Spatial-Interaction Models of Images," IEEE Trans. Information Theory, vol. 29, no. 1, pp. 60-72, 1983.

[11] R.M. Haralick, K. Shanmugam, and I. Dinstein, "Textural Features for Image Classification," IEEE Trans. Systems, Man, and Cybernetics, vol. 3, pp. 610-621, 1973.

[12] J.E. Besag, "Spatial Interaction and the Statistical Analysis of Lattice Systems (with discussion)," J. Royal Statistical Soc., B, vol. 36, pp. 192-236, 1974.

[13] P. Brodatz, Texture-A Photographic Album for Artists and Designers. New York: Reinhold, 1968.

[14] T. Bishop, S. Fienberg, and P. Holland, Discrete Multivariate Analysis-Theory and Practice. Cambridge, Mass.: MIT Press, 1975.

[15] D.A. Clausi, "An Analysis of Co-Occurrence Texture Statistics as a Function of Grey Level Quantization," Canadian J. Remote Sensing, vol. 28, no. 1, pp. 118,2002 